\title{
EVALUACIÓN DE LAS NECESIDADES DE IMPLEMENTACIÓN DE TECNOLOGÍAS DE INFORMACIÓN Y COMUNICACIÓN EN MUNICIPALIDADES NO CONSIDERADAS COMO CIUDADES PRINCIPALES: CASO OROPESA-CUSCO
}

\author{
Needs assessment of the implementation of information and communication technologies in \\ municipalities do not regarded as major cities: case Oropesa-Cusco \\ Lida León Núñez ${ }^{1, a, \equiv}$ \\ 1 Universidad Andina del Cusco, Cusco, Perú. \\ a Magister \\ $\equiv$ lida.leon@gmail.com
}

\begin{abstract}
Resumen
Las Tecnologías de la Información y de Comunicación (TIC's), se han convertido en una herramienta importante para el desarrollo de las organizaciones. Las municipalidades, como organizaciones sociales y órganos de gobierno local, no son ajenas al desarrollo que proveen las TIC's y basadas en necesidades de atención de servicios, han logrado implementar servicios tecnológicos con el entendimiento de que las TIC's permiten proporcionar una gran cantidad de servicios que pueden transformar a una comunidad aislada y reducir brechas entre los ciudadanos de un lugar y otro. Sin embargo, la forma en que han sido implementadas requiere un análisis para determinar si efectivamente están logrando su objetivo. Esta investigación describe un diagnóstico de cómo han sido implementadas los servicios que usan las tecnologías de información y comunicación en municipalidades no consideradas como ciudades principales y conocer el estado en que se hallan para que a partir de ello, se desarrolle una propuesta innovadora en el uso de las TIC's, en municipalidades que tienen esta clasificación, bajo un enfoque cualitativo que nos permita obtener la información necesaria para elaborar propuestas sustentadas en la praxis de la investigación aplicada.

Palabras claves: TIC's, Municipalidades, Diagnostico, implementación de proyectos.

Abstract

Information and Communication Technologies (TIC's) have become an important tool for the development of organizations. The municipalities, as social organizations and local government, are not alien to the development provided by TIC's and based on service needs, they have managed to implement technological services with the understanding that TIC's allow to provide a large number of services that they can transform an isolated community and reduce gaps between citizens from one place to another. However, the way in which they have been implemented requires an analysis to determine if they are effectively achieving their objective. This research describes a diagnosis of how services using information and communication technologies have been implemented in municipalities not considered as main cities and to know the state in which they are located so that, based on this, an innovative proposal for the use of ICT in municipalities that have this classification, under a qualitative approach that allows us to obtain the necessary information to develop proposals based on the practice of applied research.

Keywords: TIC's, Municipalities, Diagnosis, project implementation
\end{abstract}

Citar como: León, L. (2018). Evaluación de las necesidades de implementación de tecnologías de información y comunicación en municipalidades no consideradas como ciudades principales: caso Oropesa-Cusco. Rev Yachay, 7(1),362-366.

Recibido: 16-09-2018: Acentado 07-12-2018

\section{Introducción}

La necesidad de implementar y usar de servicios soportados por las tecnologías de información y comunicación denominadas TIC's; se ha convertido en un recurso básico en las sociedades en los últimos años, cada persona u organización, sea pública o privada, necesita comunicar, procesar, enviar y recibir información para el desarrollo de sus actividades. Muchas organizaciones han incorporado las TIC's en sus actividades diarias a fin de lograr mejorar el desarrollo de sus actividades y que estas permitan el cumplimiento de sus objetivos.

Las municipalidades han incorporado el uso de TIC's en el desarrollo de sus procesos, probablemente por un tema de cumplimiento y de necesidad, y que luego los gobiernos nacionales han desarrollado esfuerzos y han establecido algunas estrategias de cómo se deben incorporar el uso de las TIC's en organizaciones públicas. Las organizaciones gubernamentales como las municipalidades, son unidades 
territoriales y administrativas básicas del Estado y cuyo principio es promover el desarrollo de sus habitantes tal como lo señala la Ley Orgánica de Municipalidades del Perú; sin embargo debemos entender ¿cómo es que este esfuerzo se ha plasmado en estas municipalidades?, ¿o cómo estas organizaciones, especialmente en aquellas que son consideradas del tipo de menos de 500 habitantes urbanos han logrado la implementación y el uso de las TIC's?. Considerando que en muchas de estas zonas no cuentan con los servicios y las condiciones básicas que estas tecnologías necesitan para su implementación y posterior uso.

Las Municipalidades haciendo uso de su autonomía política y administrativa han implementado sus servicios bajo un criterio y conocimiento de TIC's, propio de cada gobernante, y por la necesidad de procesar, enviar y recibir información para el desempeño de sus actividades, estos han sido desarrollado con planes provisionales o improvisados para lograr el uso de las TIC's, en muchos de estos municipios estos servicios no están implementados y siguen usando procesos manuales para el apoyo de la gestión municipal. Se sabe que las tecnologías de información y comunicación permiten disminuir la brecha entre los pueblos y el hecho de contar o no contar con recursos tecnológicos puede constituir una pérdida de oportunidades de crecimiento y desarrollo para la municipalidad y sus habitantes. Entonces muchas de estas organizaciones, han tenido que adaptarse a las condiciones de recurso económico, talento humano, condiciones geográficas y otros y lograr la implementación de los servicios que proveen las TIC's.

La implementación de proyectos para incorporar el uso de tecnologías de información y comunicación en las municipalidades, se ha vuelto inminente, especialmente en las municipalidades provinciales y distritales de la capital de departamentos con el objetivo de apoyar a la gestión municipal en la toma de decisiones. ¿Pero qué pasa con municipalidades denominadas pequeñas?, aquellas que no se encuentran en ciudades principales como es el caso de Oropesa o Andahuaylillas o de otras que tienen la misma clasificación.

Las Municipalidades como es el caso de Oropesa, probablemente por falta de un adecuado conocimiento de las TIC's, no ha implementado servicios tecnológicos adecuados, confiables y seguros; han desarrollado planes improvisados, sin normas técnicas de seguridad y continuidad de sus servicios e incluso no se ha previsto la contratación del personal que se haga cargo del mantenimiento y gestión de las TIC's con que cuenta la municipalidad. La implementación de proyectos tecnológicos en este tipo de municipalidades demanda un análisis crítico y un conocimiento previo; pues el costo que implica la tecnología, con una inadecuada implementación, termina siendo perjudicial a sus presupuestos o en el peor de los casos se deja de lado su uso; por ello esta implementación debe ser analizada, ideada y aplicada a las necesidades de este tipo de municipalidades, de tal forma que su implementación sea realmente beneficioso y rentable y que apoye a la gestión municipal.

La presente investigación busca identificar las necesidades críticas que tienen estas municipalidades, como es el caso de la Municipalidad de Oropesa, en cuanto a la implementación de servicios de tecnologías de información y comunicación, para ello se debe desarrollar un adecuado diagnostico situacional interno de la municipalidad y determinar su problemática y a partir de ello definir las prioridades de implementación de servicios de tecnología de información y comunicación

\section{Materiales y Métodos}

Se realizó un estudio de tipo descriptivo - analítico, evaluándose la actual implementación de las TIC's en las municipalidades no consideradas como ciudades principales con menos de 500 habitantes urbanos, como es el caso de la municipalidad de Oropesa de tal forma se ha establecido la siguiente metodología para la obtención de información.

\section{Diagnostico Situacional de los TIC's}

Se ha realizado la obtención de la información acerca de la situación en que se encuentran implementados los servicios de tecnología de información y comunicación en la municipalidad de Oropesa; para ello se aplicó instrumentos como encuestas y entrevistas a los usuarios trabajadores, y se ha realizado una visita a las instalaciones de la municipalidad para observar la situación en que los servicios de tecnología están implementados y como hacen uso los usuarios. Se usará el Microsoft Excel para el procesamiento de esta información.

\section{Establecer los ejes de agrupación de los servicios tecnológicos}

Los servicios tecnológicos implementados en la municipalidad se han agrupado en los siguientes ejes:

1) Sistemas de Información

2) Red de datos y Comunicación

3) Equipos de Cómputo e Infraestructura Física 


\section{Resultados}

Se establecieron los ejes de agrupación de los servicios tecnológicos implementados en la municipalidad de Oropesa siendo estos:

1) Sistemas de Información

2) Red de datos y Comunicación

3) Equipos de Cómputo e Infraestructura Física

\section{Sistemas de Información}

- Se ha identificado que la municipalidad cuenta con 02 sistemas de información automatizados siendo estos: el Sistema de Tributación y el Sistema de Pagos de Agua Potable.

- Los 09 sistemas necesarios para el manejo operativo de la municipalidad Como son remuneraciones, logística, patrimonio, catastro, contabilidad, presupuesto, trámite administrativo no están automatizados se realizan de forma manual y el registro de información es almacenado en hojas de Excel, la información de catastro se almacena en el programa informático AutoCad.

- La información es dispersa y no se cuenta con información consolidada e integra, cada usuario tiene un registro de la información en función a la necesidad de uso; es decir réplicas de las hojas de Excel en cada área.

- No se cuenta con un sistema de seguridad o respaldos de información; cada usuario tiene la información en su máquina y constituye la única fuente de su información.

- No cuenta con un portal web institucional que inserte a la municipalidad en el mundo digital a fin de que difunda información de interés.

- Se tiene una cuenta de Facebook que no es alimentada constantemente, la última publicación corresponde al año 2014.

\section{Red de Datos y Comunicación}

- $\quad$ No se cuenta con una red de datos segura, el $40 \%$ de las conexiones están expuestas a la manipulación del usuario y del personal de servicio técnico que es externo a la municipalidad.

- $\quad$ No se cuenta con un software o equipos que filtren o restrinjan las páginas de internet a las que el usuario accede.

- Se ha observado que los usuarios que cuentan con el servicio de internet hacen uso de youtube en horas de trabajo, consumiendo la mayor cantidad de ancho de banda de la línea de internet.

- 10 Usuarios de la municipalidad que representa el 29\% del total de usuarios no cuenta con servicio de internet.

- $\quad 3$ usuarios no tienen su equipo conectado a la red de datos por lo tanto su trabajo se basa en aplicaciones de escritorio.

- Se ha observado que la línea de internet es una extensión de red de una cabina de internet próxima a la municipalidad cuyo cable cuelga por el techo de la municipalidad.

- Cada usuario usa su cuenta personal de correo electrónico para la comunicación de temas institucionales entre ellos y con terceros.

\section{Equipos de Cómputo e Infraestructura Física}

- $\quad$ No se cuenta con un servidor de aplicaciones y de datos que centralicen los procesos y los datos de las 02 aplicaciones automatizadas existentes.

- No se cuenta con un ambiente físico apropiado para proteger el equipo donde reside el sistema de información tributaria y de pagos; se observa una ventana de vidrio hacia la calle que genera riesgo de intrusos.

- De las 10 impresoras identificadas solo 2 trabajan en red; las demás están instaladas en el equipo del usuario que requiere mayor cantidad de impresión y cuando así otro usuario lo requiere se cambia el cable de la impresora de un equipo a otro.

- Los 25 equipos de cómputo no cuentan con modo de respaldo de información que generan y almacenan en sus equipos.

- Los respaldos de la base de datos de los 2 sistemas automatizados residen en la misma máquina donde se encuentran las aplicaciones instaladas.

\section{Discusión}

Los resultados de esta investigación, nos indican que los servicios implementados y que usan tecnologías de información y comunicación en municipalidades no consideradas como ciudades principales como es el caso de Oropesa-Cusco; requieren especial atención de tal forma que estos se mejore, cambie u optimice dichos servicios. El proceso de descentralización de los gobiernos locales necesita una respuesta institucional que no esté basada en el criterio de un gobernante o funcionario; sino de especialistas y conocedores de TIC's.

Actualmente el Estado Peruano a través de la Oficina de Gobierno Electrónico -ONGEI ha desarrollado el Plan Nacional de Gobierno Electrónico 2013-2017, donde ha definido los siguientes objetivos estratégicos: 
OE1: Lograr el desarrollo y la prestación de mejores servicios TIC para la sociedad, a través de la Interoperabilidad entre las entidades del Estado, el sector privado y la sociedad civil.

OE2: Acercar el Estado al ciudadano a través de mecanismos que aseguren el acceso oportuno e inclusivo a la información y una participación ciudadana como medio para aportar a la gobernabilidad y transparencia de la gestión del Estado

OE3: Garantizar la integridad, confidencialidad y disponibilidad de la información pública mediante mecanismos de seguridad de la información gestionada.

OE4: Fomentar la inclusión digital de todos los ciudadanos, especialmente a los sectores vulnerables, a través de la generación de capacidades y promoción de la innovación tecnológica, respetando la diversidad cultural y el medio ambiente.

OE5: Proponer y adecuar el marco legal, a fin de asegurar su cumplimiento para el despliegue del Gobierno Electrónico en el marco del desarrollo de la Sociedad de la Información.

Así mismo uno de los proyectos vinculados al objetivo OE1 y OE2 es el "Proyecto de la Red Dorsal de Fibra Óptica" que busca facilitar el acceso a los servicios de telecomunicaciones en todo el territorio peruano especialmente en las áreas rurales y lugares de preferente interés social del área de intervención del Perú; este proyecto constituye una oportunidad para este tipo de municipalidades; en caso de su implementación; de acceder a internet a un costo cero, de tal forma que su interconectividad y su inclusión en el mundo digital sea real, viable y económico.

\section{Conclusiones}

El diseño de esta investigación es denominado descriptivo- analítico porque el objetivo es conocer y describir la situación actual en que se encuentran implementados los servicios que usan tecnologías de información y comunicación y es analítica porque se busca comprender y resolver problemáticas específicas de una organización gubernamental como es el caso de las municipalidades que son no consideradas como ciudades principales. De tal forma que se pueda proporcionar la información que pueda aportar a desarrollar proyectos de mejora de servicios que usan tecnologías de información y comunicación, así como los procesos y reformas estructurales. Según Sandín (2003) quien señala que este tipo de investigación pretende, esencialmente, propiciar el que las organizaciones, como son estas municipalidades, tengan un cambio de propuesta al momento de destinar recursos económicos para la implementación de servicios que usan TIC's y que viene por el desarrollo previo de un plan estratégico de implementación de tecnologías de información de tal forma que se ajuste a las necesidades, presupuestos y objetivos y que las gestores tomen conciencia del papel determinante que tienen para decidir que tecnologías de información y comunicación deben usar e implementar.

\section{Agradecimientos}

Agradecimiento a los colaboradores del municipio de Oropesa quienes han brindado de forma transparente la información recogida para la presente investigación.

\section{Referencias Bibliográficas}

Ortega, V. V. (2013). Gobierno electrónico y ciudadanía digital para zonas rurales de Perú. Dialnet. 194.

Quinde Li Say Tan, M. J. (2016). Las TIC en el Perú desde el desarrollo sostenible: una propuesta para las zonas rurales. Universidad de Piura.

Batista, C. (2003). TICs y Buen Gobierno: La contribución de las Tecnologías de la Información y la Comunicación al Gobierno Local en América Latina. Brasilia: Universidad de Brasilia.

Bossio, J. F., López Velarde, J., Saravia, M., \& Wolf, P. (2005). Desarrollo rural y tecnologías de información y comunicación. Lima (Peru). GTZ.

Bustamante, R., Burneo, Z., \& Alvarado, M. (2009). Usos efectivos y necesidades de información para el desarrollo de estrategias apropiadas para proyectos TIC en el área rural. Informe final. Consorcio de investigación económica y social.

Domínguez A. Roció del Pilar, Málaga V. Sandra, Panez Ch. Livia Nelly (2016). Importancia del desarrollo de iniciativas de responsabilidad social empresarial mediante el uso de las TIC para el desarrollo de las zonas rurales del Perú. Universidad Peruana de Ciencias Aplicadas (UPC). Retrieved from http://hdl.handle.net/10757/621724

Electrónico, G. (2015). Plan Nacional de Gobierno Electrónico. Obtenido de http://www. gobiernoelectronico. gob. ec.

Gil, D. J. G., García, C. M. A., \& Álvarez, M. (2011). Desarrollo de comunidades rurales con TIC. In La práctica educativa en la Sociedad de la Información: Innovación a través de la investigación. Editorial Marfil.

Gómez, P. (2008). La brecha digital, brecha social. Los recursos humanos en el desarrollo y la capacitación a través del aprendizaje digital (elearning). Gazeta de Antropología. 
Guerrero Chanduví, D. A. M., \& Quinde Li Say Tan, M. J. (2011). Las TICs en el Perú

desde el desarrollo sostenible: una propuesta para las zonas rurales. In XV International Congress on Project Engineering. AEIPRO, Huesca, España. 08 de julio de 2011 (pp. 1655-1668). Universidad de Piura. Facultad de Ingeniería. Área Departamental de Ingeniería Industrial y de Sistemas. Proyectos.

Kanashiro, L. L. (2010). Implicancias del uso de las tecnologías de la información y comunicación en municipios rurales: un estudio de caso en Ayacucho, Perú.

Lozada, J. (2014). Investigación Aplicada: Definición, Propiedad Intelectual e Industria. Cienciamérica.

Quiroga, D. V. (2009). Estudio de uso y aplicaciones de las tecnologías de información y comunicación de autoridades y funcionarios en dos municipios rurales del Perú: recomendaciones para la implementación de gobierno electrónico en municipios rurales. Red Americana de Investigación en Investigación y Comunicación.

Sandín Esteban, M. P. (2003). Investigación cualitativa en educación: Fundamentos y tradiciones. Madrid: McGraw Hill; Interamericana de España.
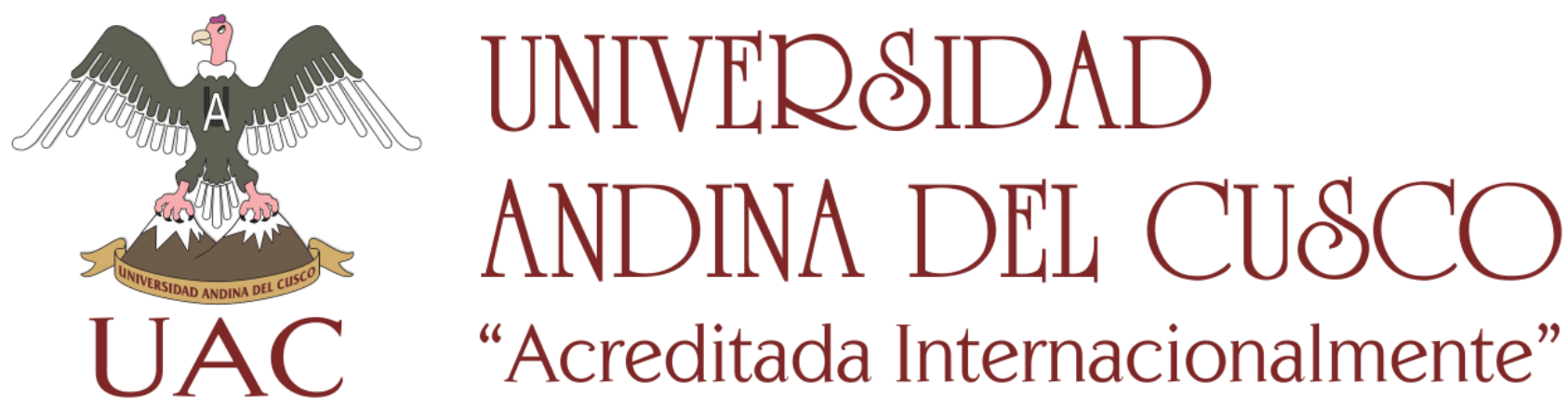